\title{
OPEN Inflammatory activation of surface molecule shedding by upregulation of the pseudoprotease iRhom 2 in colon epithelial cells
}

\author{
Anja Adelina Giese ${ }^{1,3}$, Aaron Babendreyer ${ }^{1,3}$, Peter Krappen ${ }^{1}$, Annika Gross ${ }^{2}$, Pavel Strnad ${ }^{2}$, \\ Stefan Düsterhöft ${ }^{1,3}$ \& Andreas Ludwig ${ }^{1,3 凶}$
}

The metalloproteinase ADAM17 contributes to inflammatory and proliferative responses by shedding of cell-surface molecules. By this ADAM17 is implicated in inflammation, regeneration, and permeability regulation of epithelial cells in the colon. ADAM17 maturation and surface expression requires the adapter proteins iRhom1 or iRhom2. Here we report that expression of iRhom 2 but not iRhom 1 is upregulated in intestinal tissue of mice with acute colitis. Our analysis of public databases indicates elevated iRhom 2 expression in mucosal tissue and epithelial cells from patients with inflammatory bowel disease (IBD). Consistently, expression of iRhom 2 but not iRhom 1 is upregulated in colon or intestinal epithelial cell lines after co-stimulation with tumor necrosis factor (TNF) and interferon gamma (IFNgamma). This upregulation can be reduced by inhibition of Janus kinases or transcription factors NF-kappaB or AP-1. Upregulation of iRhom2 can be mimicked by iRhom2 overexpression and is associated with enhanced maturation and surface expression of ADAM17 which then results in increased cleavage of transforming growth factor (TGF) alpha and junctional adhesion molecule (JAM)-A. Finally, the induction of these responses is suppressed by inhibition of iRhom 2 transcription. Thus, inflammatory induction of iRhom 2 may contribute to upregulated ADAM17dependent mediator and adhesion molecule release in IBD. The development of iRhom2-dependent inhibitors may allow selective targeting of inflammatory ADAM17 activities.

Inflammation and subsequent tissue repair are characterized by the increased presence of soluble mediators. These soluble mediators comprise cytokines, cytokine receptors, growth factors and adhesion molecules ${ }^{1-5}$. Several of the soluble mediators originate from limited proteolysis at a membrane-proximal, extracellular site within the membrane-anchored precursor of these mediators. This process is termed shedding and, in many cases, it involves the activity of the a disintegrin and metalloproteinase (ADAM) family members ADAM10 and ADAM17 ${ }^{6-10}$. Key substrates for ADAM17 include the tumor necrosis factor (TNF) $\alpha^{11-13}$, the transforming growth factor (TGF) $\alpha^{14}$ and the junctional adhesion molecule (JAM)- $\mathrm{A}^{8}$. These mediators are critically implicated in inflammatory and repair functions in vivo. Especially TNFa is well known to drive inflammatory bowel disease (IBD) $)^{15}$. TGF $\alpha$ contributes to epithelial tissue regeneration ${ }^{16}$ and JAM-A regulates permeability and inflammation in the intestine ${ }^{17}$. The importance of ADAM17-mediated shedding events in the intestine is indicated by analysis of transgenic mice in models of intestinal inflammation. On the one hand, hypomorphic mice with a reduced ADAM17 activity suffer from defective regeneration of epithelial cells via growth factors such as TGF $\alpha$ and breakdown of the intestinal barrier ${ }^{18}$. On the other hand, a complete, cell specific loss of ADAM17 in intestinal epithelial cells attenuates TNFa driven mucosal atrophy in a mouse model of parenteral nutrition ${ }^{19}$. Thus, ADAM17 can promote both inflammatory and regenerative processes.

The importance of ADAM17 in various pathologies as well as repair functions necessitates that the protease is tightly regulated. ADAM17 is expressed constitutively in almost all cell types and its expression can be upregulated to some degree ${ }^{20,21}$. Notably, ADAM17 is not only expressed at the cell surface but present in considerable amounts inside the cells. Moreover, overexpression is not sufficient for enhanced ADAM17-mediated shedding,

${ }^{1}$ Institute of Molecular Pharmacology, Medical Faculty, RWTH Aachen University, Pauwelsstr. 30, 52074 Aachen, Germany. ${ }^{2}$ Division of Gastroenterology and Hepatology, Department of Medicine III, University Hospital RWTH Aachen, Aachen, Germany. ${ }^{3}$ These authors contributed equally: Anja Adelina Giese, Aaron Babendreyer, Stefan Düsterhöft and Andreas Ludwig. ${ }^{\circledR}$ email: aludwig@ukaachen.de 
indicating that further posttranslational control mechanisms need to be in place before ADAM17 activity can be increased $^{22}$. First, ADAM17 is synthesized into the endoplasmic reticulum (ER) as an immature form in which the metalloproteinase activity is blocked by a prodomain ${ }^{23,24}$. In the ER immature ADAM17 interacts with iRhom 1 or 2 which are inactive members of the rhomboid family ${ }^{25-28}$. This interaction is required for ADAM17 trafficking to the Golgi apparatus where the prodomain of the protease is proteolytically removed, before the mature protease is then transported to the cell curface ${ }^{23}$. Additionally, iRhoms are necessary for the sustained expression and activity of ADAM17 on the cell surface ${ }^{29-31}$. Here mature ADAM17 can be activated by further mechanisms $s^{32,33}$, including conformational changes orienting the protease activity close to the membrane to cleave its substrates ${ }^{34}$.

So far, iRhom 1 and 2 appear to be specialized molecules for controlling ADAM17 maturation and surface expression. Notably, iRhom 2 is predominantly found in immune cells while iRhom 1 is expressed in most parenchymal tissue cells ${ }^{35}$. Consistent with this expression profile it was found that iRhom 2 is critically involved in immune functions in models of acute and chronic inflammation but is less important for physiologic functions of the tissue $\mathrm{e}^{33,36,37,37-39}$. By contrast iRhom 1 has been linked to critical homeostatic functions ${ }^{26,38-40}$.

We here address the question, whether iRhom 2 is only relevant in leukocytes or also of importance in parenchymal tissue cells. To this end, we investigate expression of both iRhoms in murine tissue from a model of colon inflammation as well as in human colon or intestinal epithelial cell lines. We describe cytokine driven transcriptional pathways that lead to upregulation of iRhom 2 but not iRhom 1 in vitro, and we demonstrate the consequences of this upregulation for ADAM17 maturation, surface expression and shedding of epithelial surface molecules.

\section{Materials and methods}

Antibodies and reagents. Unconjugated mouse monoclonal antibody (mab) against ADAM17 ectodomain (clone 111,633 MAB-9301), mouse mab isotype control (Clone 11,711) and mouse mab against iRhom2

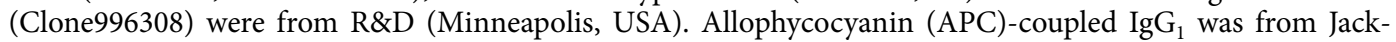
son ImmunoResearch (Hamburg, Germany). Rabbit mab against ADAM17 (C-terminus) was from Millipore (Darmstadt, Germany). Mouse mab against GAPDH (GA1R) was from Thermo Scientific (Waltham, MA USA). Peroxidase (POD)-conjugated secondary antibodies were from Jackson ImmunoResearch (Hamburg, Germany). Tumor necrosis factor a (TNFa) and Interferon $\gamma$ (IFN $\gamma$ ) were from PeproTech (Rocky Hill, USA). Nuclear factor kappa-light-chain-enhancer of activated B cells (NF- $\mathrm{kB}$ ) inhibitor Bay11-7082 and Activator protein 1 (AP-1) inhibitor SR 11,302 were from Santa Cruz Biotechnology (Dallas, USA) and JAK 1 and 2 inhibitor Baricitinib was from Selleck Chemicals (Houston, USA).

Animal tissues. The colitis experiments with 10 weeks old $\mathrm{C} 56 \mathrm{Bl} 6$ mice had been performed as previously described $^{41}$ and reversely transcribed cDNA was provided for mRNA expression analysis. In brief, mice were infected with $1 \times 10^{9} \mathrm{C}$. rodentium and sacrificed $14 \mathrm{~d}$ after infection. In another series of experiments, mice treated for a short term with dextran sodium sulfate (DSS) were given drinking water with $1.6 \%$ DSS for $4 \mathrm{~d}$ and sacrificed on day 4. Long term treated mice received drinking water containing $1.6 \%$ DSS for 5 days, followed by 2 days of tap water and then sacrificed on day 7. The animal experiments were approved by the state of North Rhine-Westphalia in Germany and the University of Aachen animal care committee and were conducted in compliance with the German Law for Welfare of Laboratory Animals and with the the ARRIVE guidelines.

Bioinformatic analyses. Public transcriptomic mRNA expression data of human samples generated by Affymetrix Human Genome U133 Plus 2.0 Array were obtained from a variety of public repositories and analyzed with the tool GENEVESTIGATORv8 suite (Nebion AG, Zürich, Switzerland) ${ }^{42}$.

The promotor region of iRhom 2 was narrowed down to -1500 bp TSS +500 bp by identifying CpG islands upstream of the transcription start site using the UCSC Genome Browser (UCSC, Santa Cruz, USA) ${ }^{43}$. The analysis of potential binding sites within this region was performed with CiiiDER(Hudson Institute of Medical Research, Melbourne, AUS) ${ }^{44}$.

Cell culture, transduction, cytokine stimulation, inhibitor treatment and transient transfection. The human epithelial colorectal adenocarcinoma cell lineHT-29 (ATCC HTB-38), the human intestinal subclone TC7 of Caco-2 cells (ATCC HTB-37) and the lung adenocarcinoma cell line A549 (ATCC CCL185) were cultured in DMEM medium with 10\% FCS and 1\% Penicillin/Streptomycin.

HT-29 cells were transduced to express iRhom2. For overexpression, cDNA encoding iRhoms (TransOMIC Technologies Inc., Huntsville, USA), was amplified by PCR and inserted into the viral expression vector pLVXIRES-Puro. Insertion was performed with the restriction enzymes EcoRI and NotI for iRhom $2 \mathrm{cDNA}$, and EcoRI and XhoI for iRhom 1 cDNA. Recombinant lentiviruses were produced as described before ${ }^{45}$. For transduction $1 \times 10^{5}$ cells were seeded into six-wells, and concentrated lentivirus preparation $(5 \mu \mathrm{l})$ was added directly after seeding. To enhance the transduction efficiency, polybrene $(8 \mu \mathrm{g} / \mathrm{ml}$, Sigma) was added. After $24 \mathrm{~h}$, cells were washed and further cultivated in complete cell culture medium for another $24 \mathrm{~h}$. Transduced cells were selected by cultivation in medium containing Puromycin $(100 \mathrm{ng} / \mathrm{ml})$ and kept in selection-medium until treatment.

For stimulation experiments, cells were grown in six-wells at a density of $2.5 \times 10^{5}$ for $24 \mathrm{~h}$. Then, TNFa or IFN $\gamma$ or a combination of both (each at concentration of $10 \mathrm{ng} / \mathrm{ml}$ ) were added and the cells were incubated for the indicated time periods before they were cooled on ice for further analysis.

For inhibitor treatment, cells received either Baricitinib $(1 \mu \mathrm{M})$, Bay11-7082 $(3 \mu \mathrm{M})$, or SR 11,302 $(3 \mu \mathrm{M}) 1 \mathrm{~h}$ prior to stimulation with TNFa and/or IFN $\gamma$. 
Transient transfection with a plasmid pcDNA 3.1 with TGFa N-terminally fused to an alkaline phosphatase was performed with Lipofectamine 3000 (Thermo; L3000015) following the manufacturer's protocol (Lipofectamine $^{\mathrm{TM}}$ Reagent protocol).

Flow cytometric analysis. Cells were stained with anti-ADAM17-ectodomain antibody ( $1 \mu \mathrm{g} / \mathrm{ml})$ in PBS supplemented with $0.2 \%$ BSA for $1 \mathrm{~h}$ on ice. Isotype control for IgG1, was used in parallel. Detection of bound antibodies was performed using APC-conjugated anti-mouse antibody (1:500). The fluorescence signal was then analyzed by flow cytometry (LSRFortessa, BD Biosciences, Heidelberg, Germany) and the median fluorescence intensity (MFI) was determined with FlowJo 8.7.3 software (Tree Star, Inc., Ashland, USA) as quantitative parameter. The unspecific MFI determined for the isotype control was subtracted from the total MFI to obtain the specific MFI for ADAM17 surface expression.

Quantitative PCR analysis. The mRNA expression levels of ADAM17, ADAM10, iRhom1 and iRhom2 were measured by quantitative real-time PCR and normalized in cell culture samples to the mRNA expression level of glyceraldehyde-3-phosphate dehydrogenase (GAPDH) and in murine tissue samples to the mRNA expression level of ribosomal protein $\mathrm{L} 7(\mathrm{~mL} 7)$. The general procedure has be described previously ${ }^{46}$. RNA of murine tissue samples was extracted using RNeasy Kit (Qiagen, Hilden, Germany). RNA extraction of cell culture samples was performed using Extractme Total RNA Kit (blirt, Gdansk, Poland). Both types of RNA samples were quantified (NanoDrop, Peqlab, Erlangen, Germany). RNA (250 ng) was reverse transcribed using RevertAid First Strand cDNA Synthesis Kit (Fermentas, St Leon-Rot, Germany) according to manufacturers' protocols. PCR reactions were then performed in duplicates of $10 \mu \mathrm{l}$ volume containing $1 \mu \mathrm{l}$ of cDNA template, $5 \mu \mathrm{l} 2 \times$ SYBR Premix Ex Taq II (Takara, Fitchburg, WI USA), $3 \mu \mathrm{H} \mathrm{H}_{2} \mathrm{O}$ and $0.5 \mu \mathrm{M}$ forward and reverse primer. Following primers were used for murine tissue samples: $m$ Adam 17 forward aaaccagaacagacccaacg; $m A D A M 17$ reverse gtacgtcgatgcagagcaaa; $m A$ dam 10 forward agcaacatctggggacaaac; $m$ Adam 10 reverse tggccagattcaacaaaaca; miRhom 2 forward agagcgtgaagtacatcc; miRhom 2 reverse taaagtctccgagcagtcc; miRhom 1 forward ttcttcacttactggctcac; miRhom 1 reverse ttccgaagtaccgagtcc; $m L 7$ forward tggaaccatggaggctgt; $m L 7$ reverse cacagcgggaacctttttc. Following primers were used for human cell culture samples: ADAM17 forward, gaagtgccaggaggcgatta; ADAM17 reverse, cgggcactcactgctattacc; ADAM10 forward ggattgtggctcattggtgggca ADAM10 reverse actctctcggggccgctgac, iRhom 2 forward cgattgacctgatccacc, iRhom 2 reverse caaagtctccgagcagtcc, iRhom 1 forward gacagcccacatctcttcac, $i R h o m 1$ reverse tccttgctcactccaaaccca. GAPDH forward, ccagccccagcgtcaaaggtg; GAPDH reverse, agggccgatcatggagtctt. All PCR reactions were run on a CFX Connect Real-Time PCR Detection System (Bio-Rad) with the following protocol: 40 cycles of $10 \mathrm{~s}$ denaturation at $95^{\circ} \mathrm{C}$, followed by $10 \mathrm{~s}$ annealing at the indicated temperature (mAdam17 $\left(57^{\circ} \mathrm{C}\right)$; mAdam10 $\left(57^{\circ} \mathrm{C}\right)$; miRhom2 $\left(60^{\circ} \mathrm{C}\right)$; miRhom1 $\left(60^{\circ} \mathrm{C}\right)$; mL7 $\left(55^{\circ} \mathrm{C}\right)$; $\operatorname{ADAM} 17\left(55^{\circ} \mathrm{C}\right)$; $\operatorname{ADAM} 10\left(61^{\circ} \mathrm{C}\right)$; iRhom $2\left(58^{\circ} \mathrm{C}\right)$; iRhom $1\left(56^{\circ} \mathrm{C}\right)$; GAPDH $\left(66^{\circ} \mathrm{C}\right)$; L7 $\left(60^{\circ} \mathrm{C}\right)$ ) and $15 \mathrm{~s}$ amplification at $72^{\circ} \mathrm{C}$. PCR efficiency was determined from the uncorrected RFU values using LinRegPCR version 2020.0 (11). Relative quantification was performed with the CFX Maestro Software 1.1 (Bio-Rad).

Western blotting. The general procedure of Western Blot detection of ADAM17 and iRhom 2 has been described previously ${ }^{46}$. Samples from cell lysates were incubated in SDS sample buffer (250 mM Tris $\mathrm{HCl}$ ( $\left.\mathrm{pH} 6.8\right)$, $50 \%(\mathrm{w} / \mathrm{v})$ glycerol, $10 \%(\mathrm{w} / \mathrm{v})$ SDS, $0.1 \%$ bromophenol blue and 5\% $\beta$-mercaptoethanol) for 30-45 min at room temperature and subjected to SDS-polyacrylamide gel electrophoresis using 10-12.5\% Tris-glycine gels. Proteins were transferred onto polyvinylidene difluoride membranes (Hybond-P, Amersham). Membranes were blocked with $5 \%(\mathrm{w} / \mathrm{v})$ non-fat dry milk in tris buffered saline with $0.05 \%$ Tween for $1 \mathrm{~h}$ and probed with primary antibodies against ADAM17 $(1 \mu \mathrm{g} / \mathrm{ml})$, iRhom $2(1 \mu \mathrm{g} / \mathrm{ml})$ or GAPDH $(1 \mu \mathrm{g} / \mathrm{ml})$ for $1 \mathrm{~h}$ or overnight at $4{ }^{\circ} \mathrm{C}$ followed by incubation with HRP-coupled secondary antibodies (diluted 1:20.000). Equal loading and transfer of proteins to the membrane was verified by detection of GAPDH using a specific monoclonal antibody. After addition of chemiluminescence substrate (ECL advanced, Amersham), signals were recorded using a luminescent image analyzer LAS3000 and Multi Gauge 3.0 software (Fujifilm, Tokyo, Japan) and quantified by using the open-source Image Studio Lite software (LI-COR Biosciences). ADAM17 was detected as two protein bands representing the mature form of ADAM17 (mADAM17) and the proform of ADAM17 (pADAM17) of 100 and $130 \mathrm{kDa}$, respectively. iRhom 2 was detected as one band of $\sim 90 \mathrm{kDa}$ and GAPDH as one band of $\sim 37 \mathrm{kDa}$. Densitometric analysis was performed with Image Studio Lite (Li-Cor, Lincoln, NB, USA). ADAM17 total protein (tADAM17) was determined as signal density of pADAM17 and mADAM17 together. Protein levels were then expressed as signal density ratio of either tADAM17 or iRhom 2 and GAPDH or pADAM17 and mADAM17.

JAM-A and IL-8 release. Unstimulated or cytokine-stimulated cells were incubated for $24 \mathrm{~h}$. Conditioned media were harvested and cleared by centrifugation $\left(10 \mathrm{~min}, 4^{\circ} \mathrm{C} ; 16000 \mathrm{~g}\right)$. Released soluble, JAM-A (JAM-A ELISA kit, SinoBiological, Beijing, China) or IL-8 (Human IL-8/CXCL8 DuoSet ELISA, R \& D Systems, Minneapolis, USA) were quantified per ELISA essentially as recommended by the manufacturers. The chromogenic reaction was mediated by a standard procedure using $0.1 \mathrm{U} / \mathrm{ml}$ streptavidin-conjugated horseradish peroxidase (Roche, Basel, Switzerland) and the BM Blue POD substrate (Roche).

Shedding activity. Shedding activity of ADAM17 was measured by an alkaline phosphatase (AP)-based assay as described before ${ }^{47}$. In brief, $1.5 \times 10^{5}$ cells were seeded in 12 -wells and transiently transfected with the ADAM17 substrate TGFa fused to an alkaline phosphatase (AP). After $4 \mathrm{~h}$ cells were washed, and fresh complete cell culture medium was added. After another $20 \mathrm{~h}$ cells were treated with cytokines or inhibitors, or both as mentioned above. The shedding activity was assessed by measuring the AP activity in the supernatant and in cell lysates (lysis buffer: 50 mM Tris; 137 mM NaCl; 2 mM EDTA; 10 mM 1,10-Phenanthroline; pH7.5). By adding 
p-Nitrophenyl phosphate (PNPP) solution (Thermo; 37,620) the AP activity could be continuously measured at $405 \mathrm{~nm}$ with the FLUOstar Optima (BMG LABTECH, Ortenberg, Germany)). To assess the AP activity the slope (change of absorption at $405 \mathrm{~nm} / \mathrm{ms}$ ) was calculated. The amount of ADAM17 activity was calculated as PNPP substrate turnover (AP activity) in the supernatant in relation to the total turnover in supernatant plus cell lysate.

Statistics. Quantitative data are shown as mean plus SD calculated from at least three independent experiments, $\mathrm{n}$ numbers are specified in the figure legends. Statistics were performed using the generalized mixed model analysis (PROC GLIMMIX, SAS 9.4, SAS Institute Inc., Cary, North Carolina, USA) and assumed to be from either normal, lognormal or beta distribution with the day of experiment conduction as random to assess differences in the size of treatment effects across the results. Residual analysis and the Shapiro-Wilk test were used as diagnostics. In the case of heteroscedasticity (according to the covtest statement) the degrees of freedom were adjusted by the Kenward-Roger approximation. All p-values were adjusted for multiple comparisons by the false discovery rate (FDR).

Ethics approval and consent to participate. We herewith confirm that all methods were carried out in accordance with relevant guidelines and regulations.

\section{Results}

iRhom 2 but not iRhom 1 is transcriptionally upregulated in a colitis model. Tissue cells are known to express iRhom 1 under basal conditions while immune cells preferentially express iRhom $2^{26,35}$. To get an idea of the expression level in the healthy colon, we performed a bioinformatic analysis of the reported expression data for different colon epithelial tissues using GENEVESTIGATOR ${ }^{\oplus 42}$. This analysis revealed that iRhom 1 mRNA is highly expressed in these tissues under basal conditions, whereas iRhom 2 mRNA is expressed at considerably lower levels (suppl. Figure 1). Of note, ADAM17 is also highly expressed in these tissues.

To further investigate the possible regulation of both iRhoms under inflammatory conditions we used colonic tissues previously obtained from different murine colitis models to study the mRNA expression of iRhom 1 and 2. In these models colitis had been induced by either bacterial infection with C. rodentium, 4 days of DSS supply with drinking water (short-term model) or 5 days of DSS supply plus 2 days without DSS (long-term model) ${ }^{41}$. We found that mRNA expression of iRhom 2 but not iRhom 1 is increased threefold in the long-term DSS model (Fig. 1A,B). Also, ADAM17 and ADAM10 mRNA expression was upregulated almost twofold under these conditions (Fig. 1C,D). Notably neither the bacterial infection nor the short-term exposure with DSS were sufficient to increase iRhom 2 mRNA expression. Further analysis of transcriptome data from human colon tissue samples revealed that iRhom 2 is upregulated in mucosal tissue and colon epithelial cells from patients with Crohn's disease (CD) or ulcerative colitis (UC) (Fig. 1E,F).

Cytokines upregulate iRhom 2 but not iRhom1 in a gut epithelial cell line. To study further whether iRhom 2 can be upregulated in vitro we chose the human colon epithelial cell line HT-29. As stimuli we used the cytokines IFN $\gamma$ and TNFa which are well known to act as critical proinflammatory mediators during the course of colitis ${ }^{48}$. Cells were either treated with IFN $\gamma$ or TNF $\alpha$ alone or in combination. We observed a detectable basal expression of iRhom 1 and iRhom 2 in these cells (Fig. 2A,B). The iRhom 2 mRNA expression was not increased by IFN $\gamma$ or TNFa alone but was increased by a combination of both cytokines. (Fig. 2A). In contrast, iRhom 1 was not upregulated by the cytokines either alone or in combination (Fig. 2B). The expression of ADAM17 mRNA and ADAM10 mRNA were also not affected (Fig. 2C,D). Thus, IFN $\gamma$ and TNFa seem to cooperate to specifically enhance the iRhom 2 expression in gut epithelial cells.

We next studied the time kinetic of iRhom 2 upregulation in response to co-stimulation with IFN $\gamma$ and TNFa. Interestingly, the induction turned out to be transient with a sharp peek at the $6 \mathrm{~h}$ time point and at $12 \mathrm{~h}$ the expression again declined (Fig. 2E). By contrast induction of CXCL8 expression was more rapid with a peak at $1 \mathrm{~h}$ and declining thereafter. Neither the gene expression of iRhom1, ADAM17 nor ADAM10 was induced by co-stimulation over the investigated time period (suppl. Figure 2.).

Upregulation of iRhom2 leads to increased maturation and surface expression of ADAM17. Since the $6 \mathrm{~h}$ of cytokine stimulation was optimal for mRNA induction we expected that effects on the protein level should become visible sometime after $6 \mathrm{~h}$. In fact, after $24 \mathrm{~h}$ stimulation with TNFa or costimulation with IFN $\gamma$ and TNFa a significant upregulation of iRhom 2 protein expression was detectable by western blotting using a monoclonal antibody against iRhom2 (Fig. 3A,B, suppl. Figure 3A) Stimulation with IFN $\gamma$ or TNFa alone did not have a significant effect on the protein expression level. Since there exists no reliable antibody against iRhom 1 we could not investigate iRhom 1 protein expression.

The main function of iRhoms is to associate with ADAM17 and to promote its maturation and surface expression ${ }^{25,49,50,51}$. Therefore, we performed western blot analysis of cell lysates from stimulated and unstimulated cells to study whether the upregulation of iRhom 2 associates with the increased presence of mature ADAM17 compared to the proform of ADAM17 (Fig. 3A-D). In fact, co-stimulation increased the maturation level of ADAM17, but there was no significant upregulation of total ADAM17 protein expression at this time point.

Since only mature ADAM17 is transported to the cell surface ${ }^{23}$ we asked whether the increased maturation would result in increased surface expression of ADAM17. Flow cytometric analysis revealed that co-stimulation with IFN $\gamma$ and TNFa significantly increased ADAM17 surface expression (Fig. 3E,F). By contrast, stimulation with either IFN $\gamma$ or TNF $\alpha$ alone had a less pronounced effect or no effect, respectively.

To address the question whether the observed regulatory mechanism would also occur in other epithelial cell lines we performed corresponding experiments with the human intestinal epithelial subclone TC7 of Caco-2 cells 

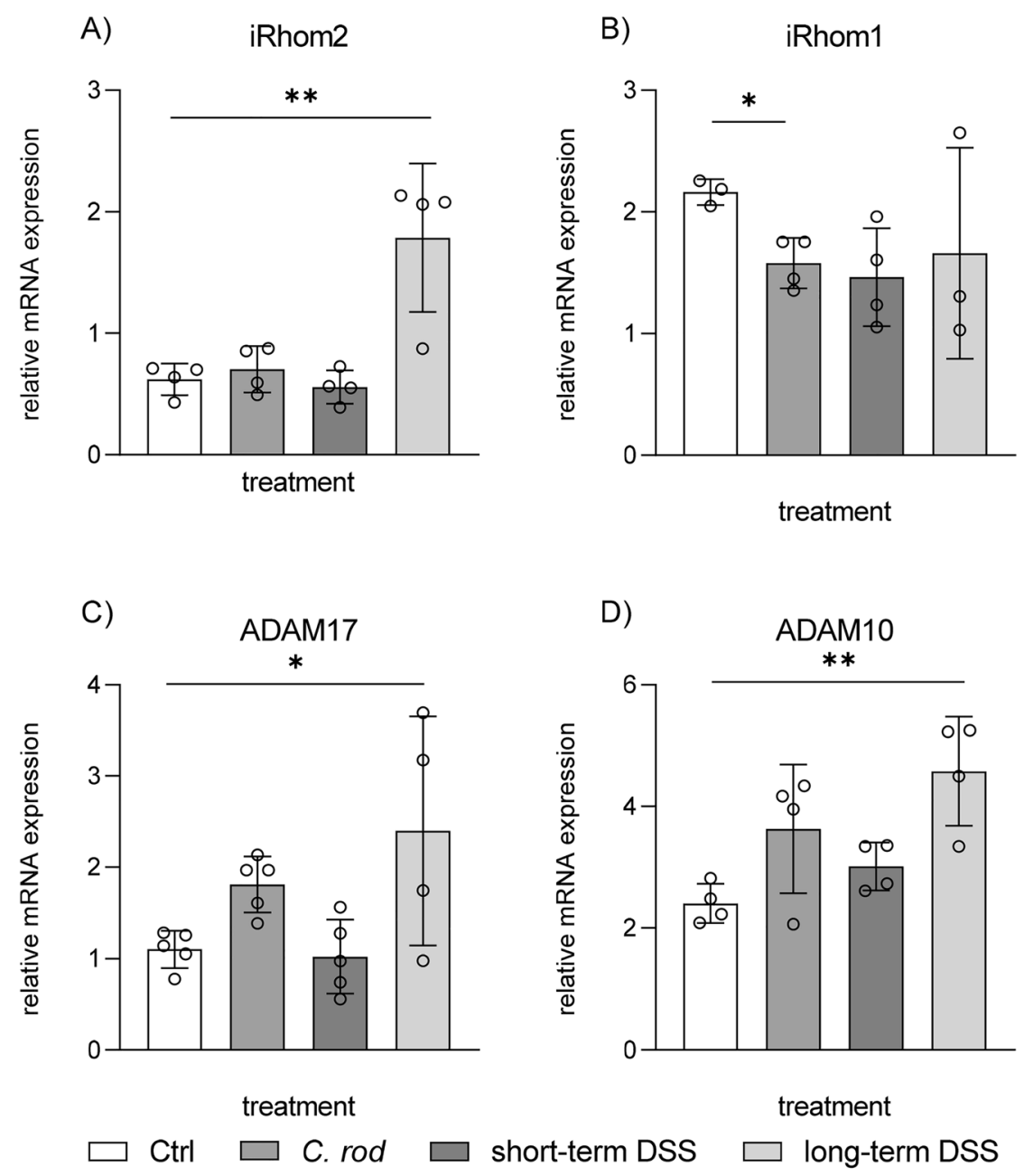

E)

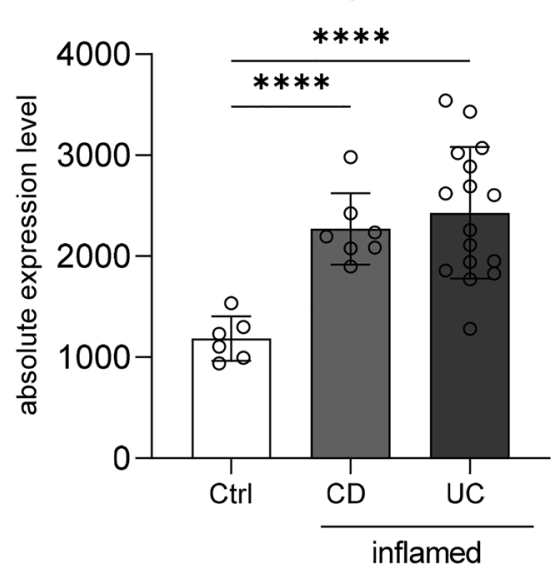

Mucosa

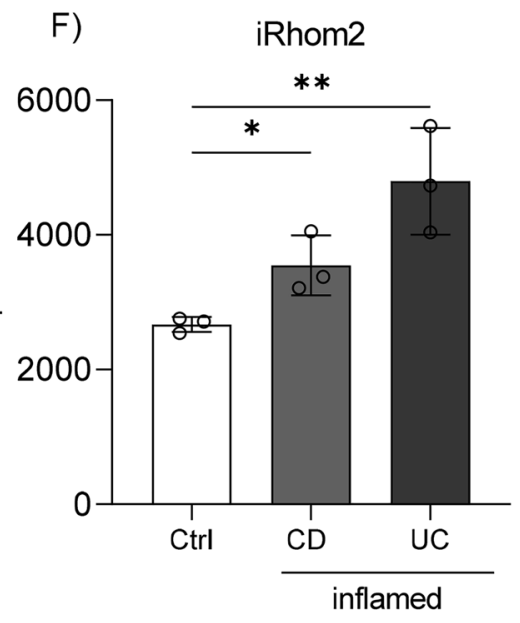

Epithelium

Figure 1. Long-term treatment with DSS leads to induced mRNA expression of iRhom 2 and ADAM17 in murine colonic tissue. (A-D) Colon tissue was used from different murine models of colon inflammation. Mice had been either infected with C. rodentium or treated with DSS in drinking water for a short-term or for long-term, respectively. Colon tissues were analyzed for relative mRNA expression of iRhom2 (A), iRhom1 (B), ADAM17 (C) and ADAM10 (D) with mL7 as reference gene by qPCR. (E-F): Analysis of public transcriptome data from human colon tissue samples revealed that iRhom 2 is upregulated in mucosal tissue (E) and colon epithelial cells (F) from patients with Crohn's disease (CD) or ulcerative colitis (UC). Data are shown as mean + SD of at least three independent experiments. Statistical differences in comparison to the control (Ctrl) are indicated by asterisks $\left({ }^{*}=\mathrm{p} \leq 0.05 ;{ }^{* *}=\mathrm{p} \leq 0.01 ;{ }^{* * *}=\mathrm{p} \leq 0.001,{ }^{* * *}=\mathrm{p} \leq 0.001\right)$. 
A)

iRhom2

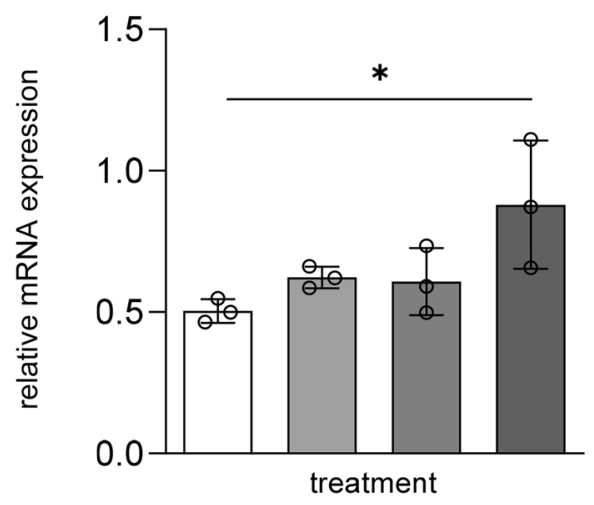

C)

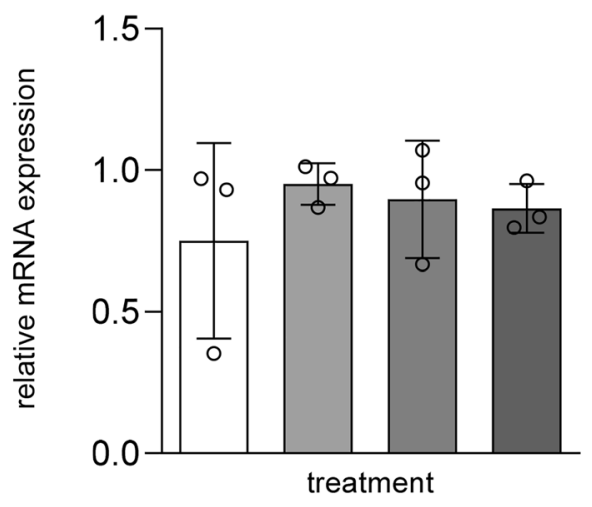

B)

iRhom1

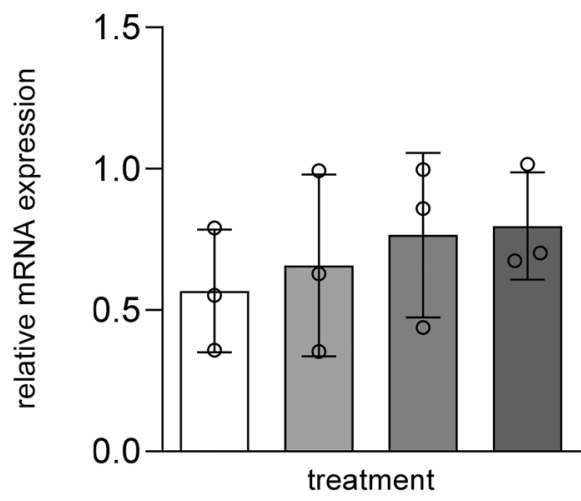

D)

ADAM10

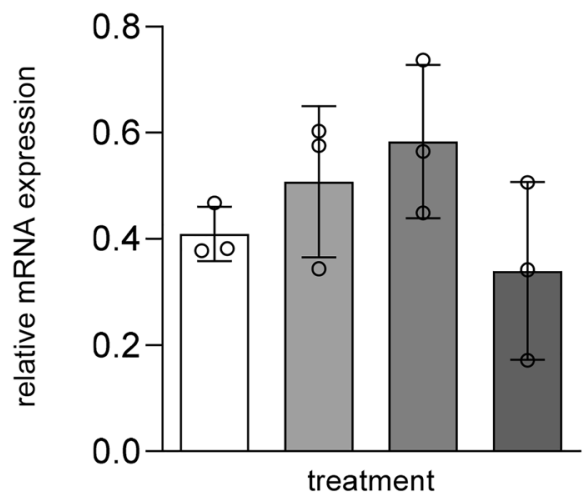

TNFa

IFNY + TNFa

E)

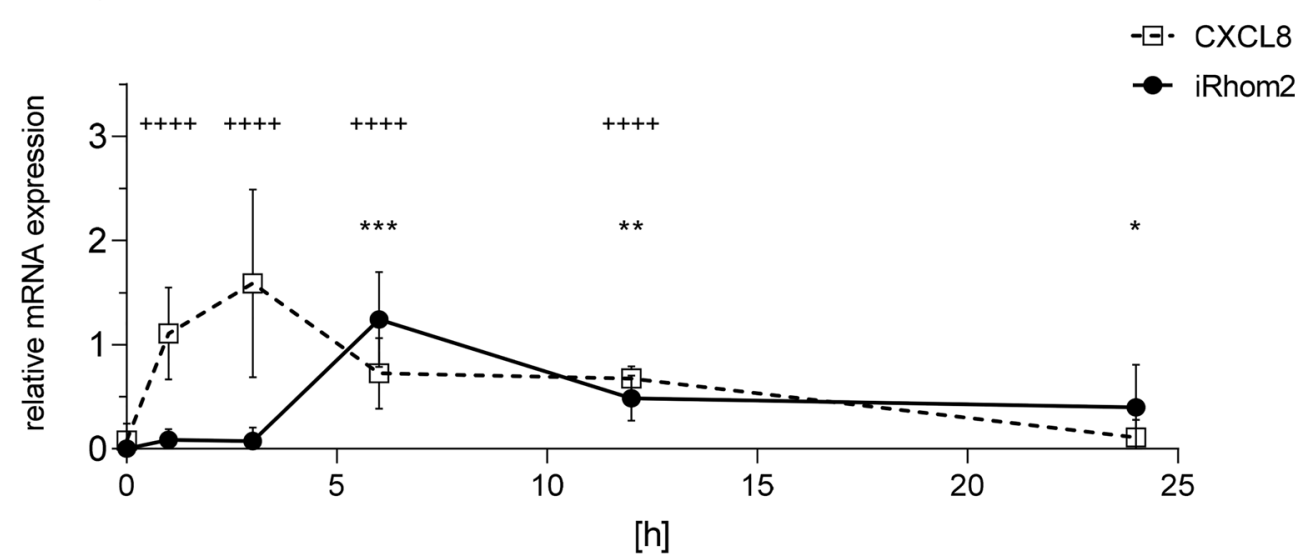

Figure 2. Stimulation with IFN $\gamma$ and TNFa leads to an induced expression of iRhom 2 in HT-29 cells. HT-29 cells were left unstimulated (Ctrl) or stimulated with IFN $\gamma$, TNFa or both (each cytokine $10 \mathrm{ng} / \mathrm{ml}$ ) for $24 \mathrm{~h}$. Afterwards, cells were harvested and analyzed by qPCR for relative mRNA expression of iRhom2 (A), iRhom1 (B), ADAM17 (C) and ADAM10 (D) with GAPDH as reference gene. HT-29 cells were stimulated with a combination of IFN $\gamma$ and TNFa (each cytokine $10 \mathrm{ng} / \mathrm{ml}$ ) for the indicated time periods. Subsequently, mRNA expression of iRhom 2 and IL-8 was analyzed by qPCR with GAPDH as reference gene. The mRNA expression shown indicates the expression of CXCL8 or iRhom2 minus the basal expression at each indicated time point (E). Data are shown as mean $+\mathrm{SD}$ of at least three independent experiments. Statistical differences in comparison to the control (Ctrl) are indicated by asterisks for iRhom and crosses for CXCL8 $\left({ }^{*}=\mathrm{p} \leq 0.05\right.$; $\left.{ }^{* *}=\mathrm{p} \leq 0.01 ;{ }^{* * *}=\mathrm{p} \leq 0.001\right)$. 
A)

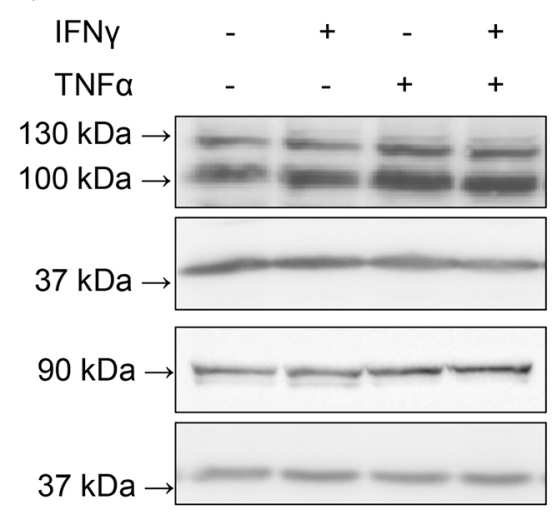

C)

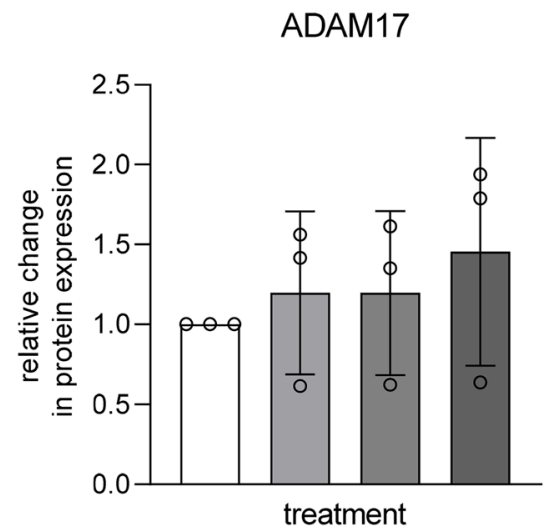

E)

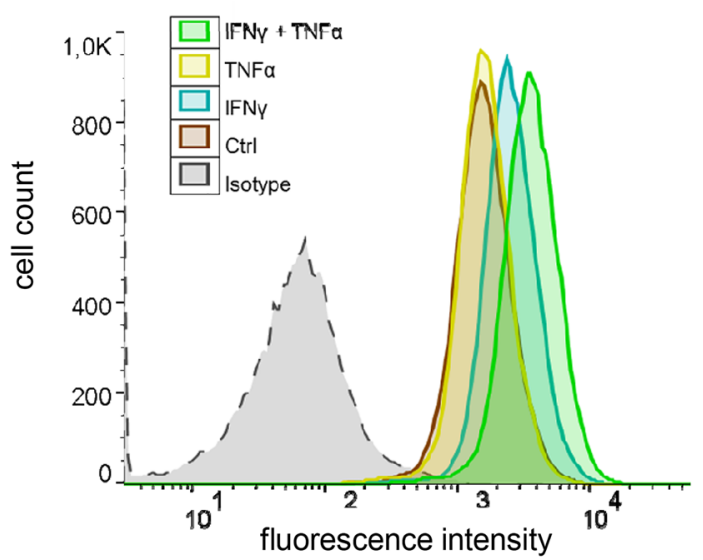

B)

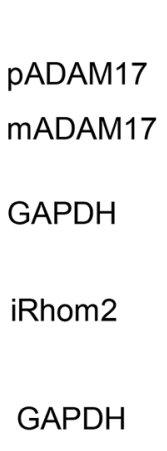

D)

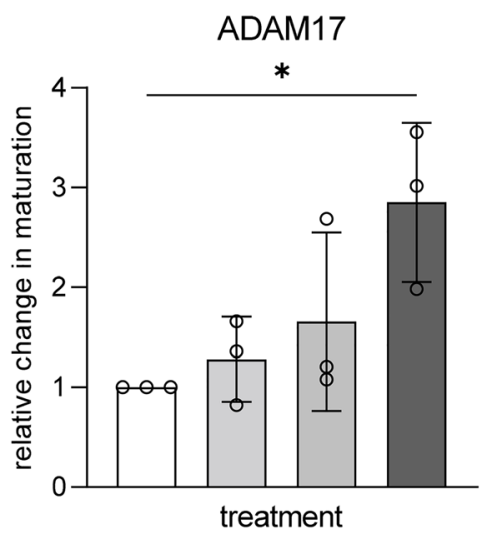

F)

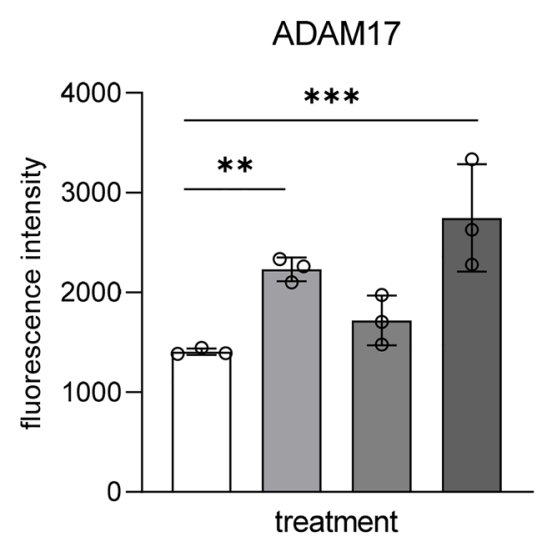

Figure 3. Co-stimulation with IFN $\gamma$ and TNFa leads to increased iRhom 2 protein expression, ADAM17 maturation and surface expression. HT-29 cells were left unstimulated (Ctrl) or stimulated with IFN $\gamma$, TNFa or both (each cytokine $10 \mathrm{ng} /$ $\mathrm{ml}$ ) for $24 \mathrm{~h}$. (A-D): Subsequently, protein levels of iRhom 2 and ADAM17 were analyzed by western blot with GAPDH as loading control. Exemplary western blots of iRhom2, ADAM17 and GAPDH are shown (A). Full western blot images are provided in suppl. (A) Protein expression of iRhom2 (B) and ADAM17 (C) as well as ADAM17 maturation (D) were quantified by densitometric analysis. ADAM17 surface expression was determined by flow cytometry (E,F). An exemplary histogram $(\mathbf{E})$ and the quantified specific median fluorescence $(\mathbf{F})$ are shown. All quantitative data are displayed as mean $+\mathrm{SD}$ of three independent experiments. Statistical differences in comparison to the control (ctrl) are indicated by black asterisks $\left({ }^{*}=\mathrm{p} \leq 0.05 ;{ }^{* *}=\mathrm{p} \leq 0.01 ;{ }^{* * *}=\mathrm{p} \leq 0.001\right)$. 
and the human lung epithelial cell line A549. As shown before for HT-29 cells, we could observe an induction of iRhom 2 but not iRhom 1 in response to co-stimulation with the cytokines IFN $\gamma$ and TNF $\alpha$ which correlated with increased maturation of ADAM17 in both Caco-2 (suppl. Figure 4) and A549 (suppl. Figure 5) cells. The upregulation of ADAM17 surface expression was also evidenced for A549 cells (suppl. Figure 5F). Thus, the observed regulatory mechanism for ADAM17 seems to occur in different types of epithelial cell lines even from different organs.

Upregulation of iRhom 2 involves signaling by NF-KB, AP-1 and JAK. Since co-stimulation with IFN $\gamma$ and TNF $\alpha$ could efficiently upregulate iRhom 2 expression we next analyzed the involvement of key signaling events that are known to be activated by these cytokines (Fig. 4). We first performed a bioinformatic binding site analysis using $\mathrm{CiiiDER}^{44}$ for the putative promoter region of iRhom 2 and recognized potential binding sites for NF- $\kappa$ B, AP- 1 and STAT1 transcription factors. In fact, there were 4 typical sites for NF- $\kappa$ B proteins, 6 for AP-1 proteins and 1 for the transcription factor STAT1 in iRhom2 (suppl. Figure 3B).

To study whether NF- $\mathrm{KB}$ or AP-1 are relevant for transcriptional induction of $\mathrm{iRhom} 2$ expression we used the pharmacological inhibitors Bay11-7082 and SR 11302, respectively. Both transcriptional pathways can be triggered by TNFa ${ }^{52,53}$, while only the NF- $\kappa$ B pathway but not the AP-1 pathway is activated by IFN $\gamma^{53,54}$. To selectively inhibit IFN $\gamma$ induced signaling we chose Baricitinib which blocks JAK activity that is directly triggered by IFN $\gamma$ receptors upon ligand binding.

We found that all inhibitors reduced the rise in iRhom 2 mRNA (Fig. 4A-C) and protein expression (Fig. 4D-F and suppl. Figure 6D-F) induced by the combined stimulation with IFN $\gamma$ and TNFa. Noteworthy, the most complete inhibition was observed with Baricitinib, while Bay11-7082 and SR 11302 lead to partial inhibition. Moreover, this inhibition was associated with decreased ADAM17 maturation (Fig. 4G-I) and reduced ADAM17 surface expression (Fig. 4J-L and suppl. Figure 7.) in cells stimulated with both cytokines.

Effects of iRhom 2 upregulation can be mimicked by iRhom 2 overexpression. Next, we asked whether overexpression of iRhom 2 or iRhom 1 would be sufficient to upregulate ADAM17 maturation and surface expression (Fig. 5.). To this end, the cells were transduced with lentivirus encoding human iRhom 1 or iRhom 2 and overexpression was controlled on the mRNA level for both iRhoms (Fig. 5A, B) and at the protein level for iRhom 2 (Fig. 5D and suppl. Figure 8.A). It was confirmed that either iRhom 1 or iRhom 2 could be overexpressed and specifically detected. Importantly, mRNA expression of neither ADAM17 (Fig. 5C) nor its close relative ADAM10 (suppl. Figure 8B) change with long-term overexpression of iRhom 1 or iRhom2. Western blot analysis for ADAM17 protein expression then revealed that overexpression of iRhom 2 enhanced maturation of ADAM17 (Fig. 5E and suppl. Figure 8A). Furthermore, flow cytometric analysis confirmed that surface expression of ADAM17 was enhanced as a consequence of this maturation (Fig. $5 \mathrm{~F}$ and suppl. Figure 8C). By contrast, overexpression of iRhom 1 did not significantly affect ADAM17 maturation nor surface expression (Fig. 5E,F). These results indicate that the effects of inflammatory iRhom 2 upregulation on ADAM17 maturation and trafficking can be efficiently mimicked by overexpression of iRhom 2 but not by overexpression of iRhom 1 .

iRhom2 upregulation or overexpression promote ADAM17 dependent shedding. To assess how changes in the expression level of iRhoms would affect ADAM17-mediated shedding we performed a shedding assay for TGFa which is a well-established substrate of ADAM17 and is not significantly shed by other related proteases ${ }^{14}$. For easy and reliable quantification of TGF $\alpha$ shedding, cells were transfected with TGFa $\mathrm{N}$-terminally fused to an alkaline phosphatase. This engineered substrate could be enzymatically detected in the cell lysate and in the supernatant. TGFa was constitutively shed in unstimulated and untransfected cells. Induction of iRhom 2 expression by co-stimulation with IFN $\gamma$ and TNFa was associated with increased TGFa shedding, which could be mimicked by overexpression of iRhom 2 but not iRhom1 (Fig. 6A). Moreover, this effect was suppressed when the transcriptional induction of iRhom 2 after co-stimulation with IFN $\gamma$ and TNFa was prevented with AP-1- (SR 11302) or JAK- (Baricitinib) inhibitors (Fig. 6B). A reduction of TGFa release was also noted with the NF $\kappa B$ inhibitor (Bay11-7082) but this did not reach significance. These data indicate that increased presence of iRhom 2 leads to increased ADAM17 activity. To finally investigate the release of an endogenous substrate we chose the junctional adhesion molecule JAM-A which is constitutively expressed by epithelial cells and is a confirmed substrate of ADAM $17^{8}$. The release of shed, soluble JAM-A into the supernatant of HT-29 cells was determined by ELISA (Fig. 6C). As previously detected for TGFa, the induced iRhom 2 expression after co-stimulation with IFN $\gamma$ and TNFa led to increased shedding of JAM-A. This could be mimicked by the overexpression of iRhom 2 but not iRhom 1 . Therefore, an increased iRhom 2 expression level clearly affects physiological ADAM17 substrates with known critical functions in maintaining cell-cell contacts in IBD ${ }^{18,31,55}$.

\section{Discussion}

The present study demonstrates that iRhom 2 but not iRhom 1 is upregulated in intestinal tissue of mice undergoing prolonged DSS induced colitis. The cytokines IFN $\gamma$ and TNFa synergistically upregulate iRhom 2 mRNA and protein expression in the colon epithelial cell line HT-29. By contrast, iRhom 1 is constitutively expressed and not further upregulated by these cytokines. The upregulation of iRhom 2 can be reduced by either inhibition of Janus kinases, NF- $\mathrm{kB}$ or AP-1. The iRhom 2 upregulation is associated with enhanced maturation and surface expression of ADAM17. These effects can be mimicked by overexpression of iRhom 2 but not by overexpression of iRhom 1 . Finally, we demonstrate that enhanced iRhom 2 expression by either cytokine stimulation or overexpression results in increased cleavage of the ADAM17 substrates TGF $\alpha$ and JAM-A. Our data therefore indicate that inflammatory iRhom 2 upregulation is instrumental to further mobilize intracellularly stored immature ADAM17 and to increase ADAM17 shedding activity. 
Bay 11-7082 (NF-KB)

SR 11302 (AP-1)

Baricitinib (JAK)

A)

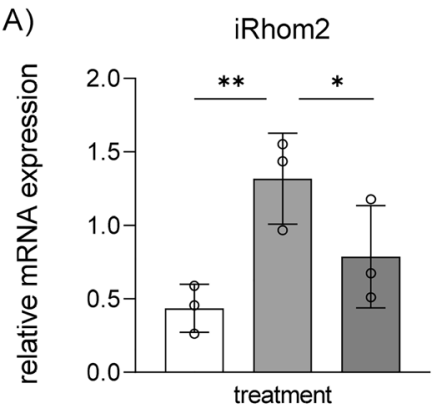

D)

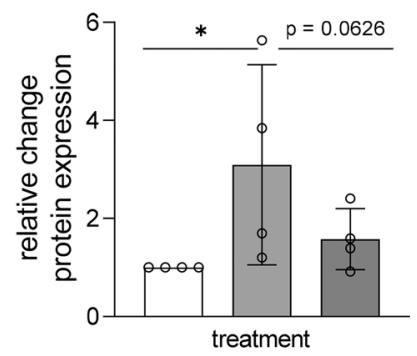

G)

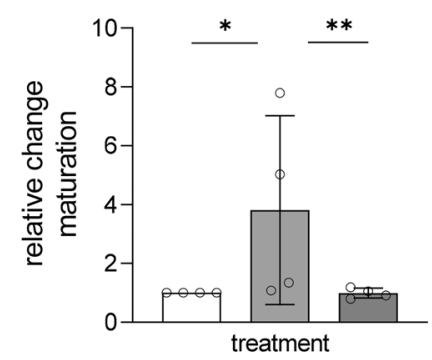

J)

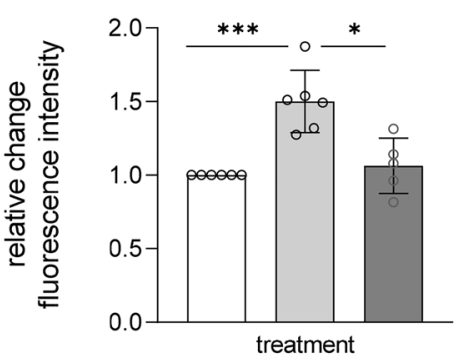

B)

iRhom2

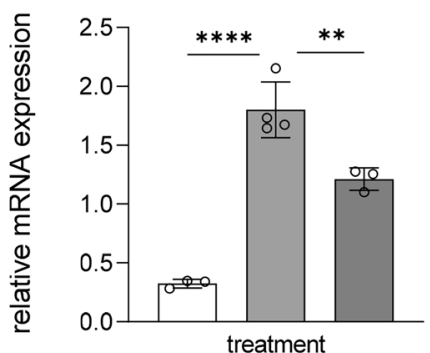

E)

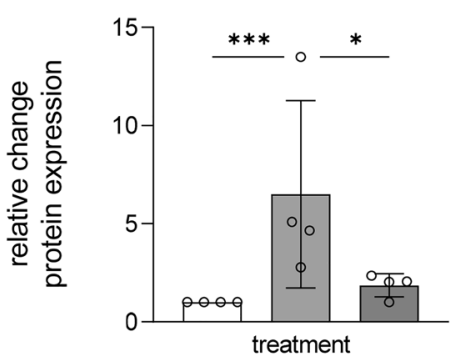

H)

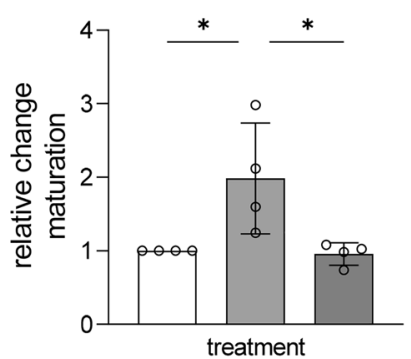

K)

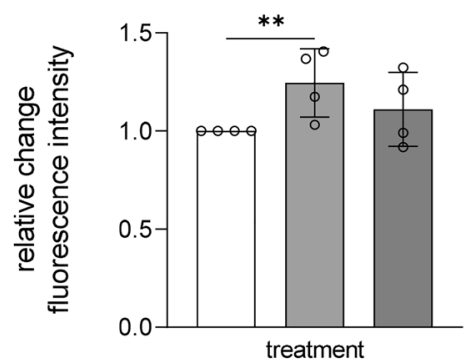

C)

iRhom2

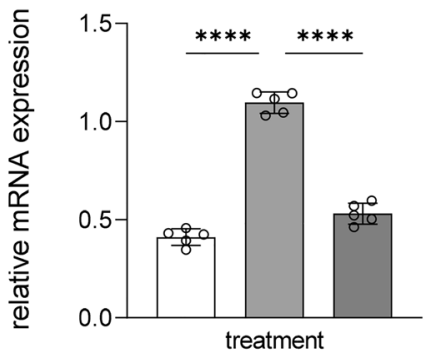

F)

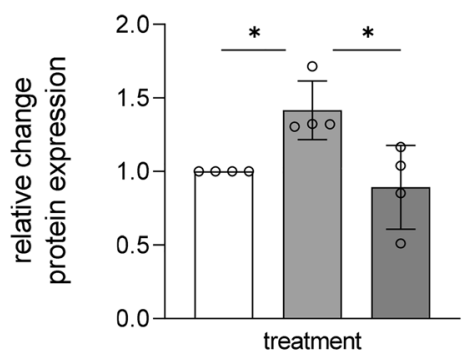

I)

ADAM17

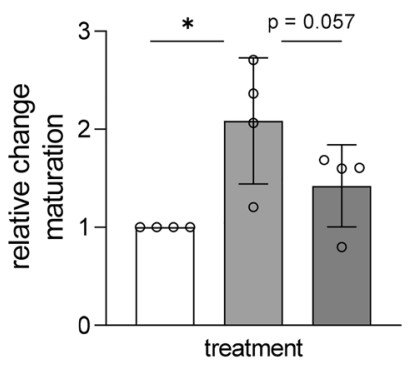

L)

ADAM17

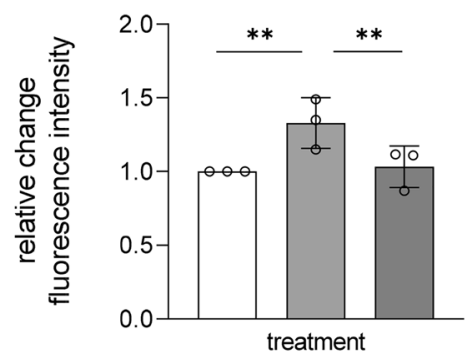

DMSO $\square$ IFNY + TNFa $\square$ Inhibitor \& IFNY + TNFa

Figure 4. Inhibition of $i$ Rhom 2 upregulation and ADAM17 regulation. HT-29 cells were treated with the NF-kB inhibitor Bay11-7082 (3 $\mu \mathrm{M})$, the AP-1 inhibitor SR 11,302 $(3 \mu \mathrm{M})$, the JAK inhibitor Baricitinib $(1 \mu \mathrm{M})$ or with the appropriate volume of vehicle control (DMSO). After $1 \mathrm{~h}$, cells were co-stimulated with IFN $\gamma$ and TNFa (each cytokine $10 \mathrm{ng} / \mathrm{ml}$ ) or left unstimulated. For analyzing mRNA of iRhom 2 by qPCR with GAPDH as reference gene (A-C), cells were stimulated for $6 \mathrm{~h}$. For the examination of protein levels of iRhom 2 with GAPDH as loading control (D-F) and maturation of ADAM17 by western blot (G-I) and ADAM17 surface expression by flow cytometry $(\mathbf{J}-\mathbf{L})$ cells were stimulated for $24 \mathrm{~h}$. Quantitative data are displayed as mean $+\mathrm{SD}$ of at least three independent experiments. Statistical differences in comparison to IFN $\gamma$ and TNFa stimulated cells are indicated by asterisks $\left({ }^{*}=\mathrm{p} \leq 0.05 ;{ }^{* *}=\mathrm{p} \leq 0.01 ;{ }^{* * *}=\mathrm{p} \leq 0.001\right)$. 
A)

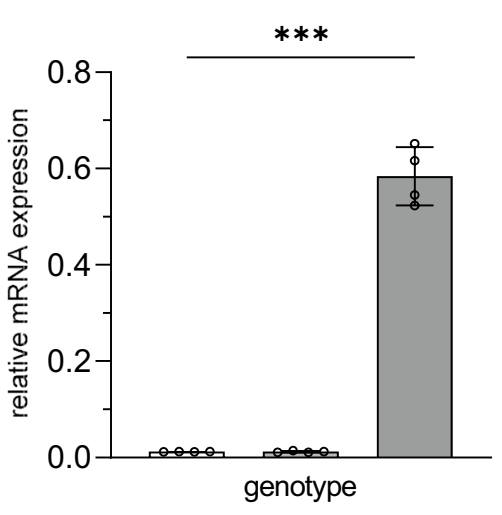

D)

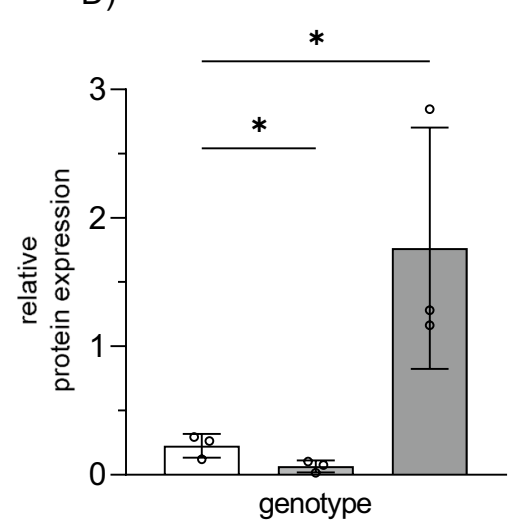

B)

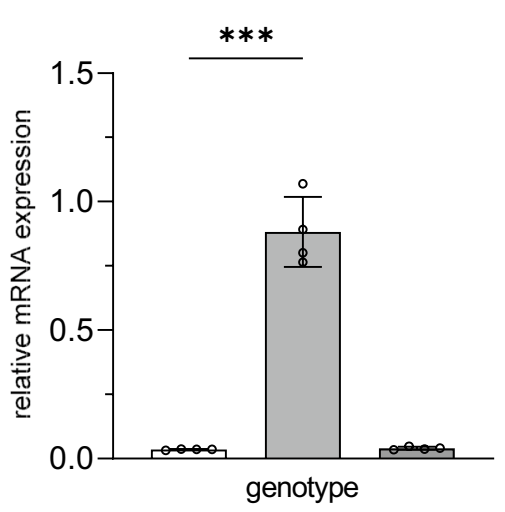

E)

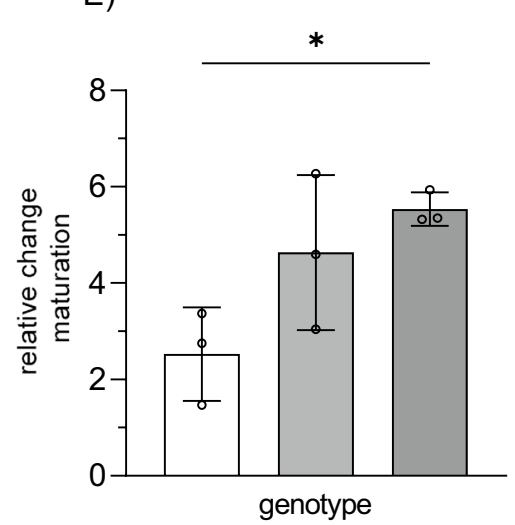

C)

ADAM17

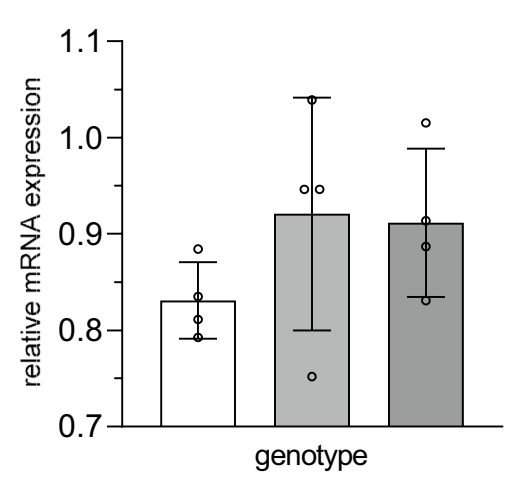

F)

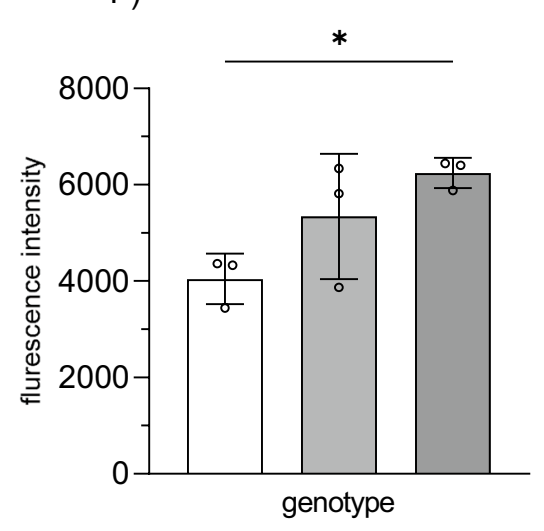

Figure 5. Effect of iRhom 2 overexpression on ADAM17 regulation. HT-29 cells were transduced with lentivirus coding for iRhom 1 (iRhom1 OE) or 2 (iRhom2 OE) or with control vector (Ctrl). After selection of transduced cells, mRNA expression of iRhom2 (A), iRhom1 (B), ADAM17 (C) was examined by qPCR with GAPDH as reference gene. Protein expression of iRhom 2 with GAPDH as loading control and maturation of ADAM17 were analyzed by western blot (D,E). ADAM17 surface expression was determined by flow cytometry (F). Quantitative data are displayed as mean $+\mathrm{SD}$ of at least three independent experiments. Statistical differences in comparison to the control (ctrl) are indicated by asterisks ${ }^{*}=\mathrm{p} \leq 0.05 ;{ }^{* *}=\mathrm{p} \leq 0.01$; $* * *=\mathrm{p} \leq 0.001)$.

Previous research has demonstrated that iRhom 2 is critical for ADAM17 maturation in immune cells ${ }^{25,31,49,36,50}$. In tissue cells under basal conditions, however, iRhom 2 is expressed at a low level and iRhom 1 seems to fulfill this function for ADAM17. Consistent with this theory, our bioinformatic analysis demonstrated that iRhom 2 is expressed at a low constitutive level in colon epithelial cells whereas iRhom 1 is present at a much higher expression level already in unstimulated cells. However, under inflammatory conditions the situation is very different. Here, iRhom 2 becomes significantly upregulated and therefore it plays a significant role for the upregulation of ADAM17 maturation, surface expression and activity in colon epithelial cells undergoing inflammatory stimulation. A regulatory mechanism which has also been demonstrated in endothelial cells after inflammatory stimulation ${ }^{46}$. Thus, our findings significantly extend the current knowledge on the specific task of 
both iRhoms for promoting ADAM17 function by demonstrating that iRhom 2 is not only relevant in immune cells but also in epithelial tissue cells under inflammatory conditions.

As explained above, iRhom 1 is expressed already in resting cells where iRhom 2 is only weakly expressed. Thus, at this stage constitutive ADAM17 maturation should be mainly due to iRhom 1. Overexpression of iRhom 1 only slightly enhances ADAM17 maturation. But this is not significant and not comparable to the effect of iRhom2 overexpression. This could be due to differences between both iRhoms in their trafficking mechanism towards ADAM17, in their affinity for ADAM17, in their stability after complex formation with ADAM17 or in their susceptibility to an unknown inhibition mechanism. Further research needs to determine whether such differences reside in the N-terminal part of iRhoms showing the lowest degree of homology between both proteins ${ }^{56}$.

IFN $\gamma$ and TNF $\alpha$ are well known to promote the development of IBD mostly by induction of inflammatory transcriptional pathways. We found that the expression of iRhom 2 is considerably upregulated by co-stimulation with these two cytokines. Interestingly, either cytokine alone has no or only weak effects on iRhom 2 expression. These data indicate a cooperation of signaling pathways of the two cytokines. Synergism of both cytokines has also been reported in DSS induced colitis ${ }^{57}$. We observed that the cooperative induction of iRhom 2 expression by IFN $\gamma$ and TNFa is almost completely switched off by blocking the JAK activity downstream of the IFN $\gamma$ receptor with Baricitinib. These results indicate the importance of JAK for inducing iRhom2-dependent ADAM17 activity in an inflammatory setting. They also indicate that this approved inhibitor routinely used for treatment of chronic inflammatory diseases is capable to limit the activity of this protease. Our bioinformatic promoter analysis indicates that both NF- $\mathrm{KB}$ and AP-1 can be involved in iRhom 2 induction and in fact this was confirmed using inhibitors for either transcription factor. Interestingly NF- $\kappa B$ can be directly switched on by IFN $\gamma^{54,58,59}$ as well as by $\mathrm{TNF}^{60,61}$. However, it seems likely that the binding of NF- $\kappa \mathrm{B}$ to the few sites is not sufficient for iRhom 2 induction and that more efficient induction occurs when the AP-1 pathway is also triggered via TNFa, thereby promoting AP-1 binding to the iRhom 2 promoter. To study this possibility in more detail and to gain more insight on the individual binding sites as well as other possible pathways, a mutational in-depth promoter analysis is necessary. In addition, posttranscriptional mechanisms may be implicated leading to enhanced presence of iRhom 2 protein. It may be possible that TNFa stimulation leads to stabilization of iRhom 2 mRNA similarly as it has been described for CX3CL1 mRNA ${ }^{62}$.

IBD is driven by several mediators of chronic inflammation and increasing evidence indicates that the impairment of the epithelial barrier function in the gut is a crucial pathogenic factor. In this setting the iRhom/ADAM17 axis may play an ambivalent role, by activating protective pathways important for regeneration as well as by inducing inflammatory signaling. Protective pathways can be triggered by the shedding of growth factors which then transactivate EGFR and by this mediate epithelial regeneration ${ }^{5,9,18,63,64,65}$. The importance of ADAM17 for this process has been demonstrated using mice with severely reduced ADAM17 expression which suffer from increased barrier disruption. In normal gut tissue, the basal presence of ADAM17 and iRhom 1 expression appears to be sufficient to maintain basal shedding activity providing growth signals for tissue regeneration. Under inflammatory conditions however, iRhom 2 upregulation would promote additional ADAM17 activity and this would generate an excess of growth. It is not known whether the basal iRhom1 expression is sufficient to cope with the increased need of tissue regeneration. Interestingly, an iRhom 2 mutation leading to reduction of function did not affect sensitivity to DSS induced weight loss, implying that iRhom 2 was physiologically redundant for the processing of EGFR ligands ${ }^{50}$.

ADAM17 is critical for the release of soluble TNFa which is a crucial driver of the inflammatory disease. In fact, by tissue specific knockout, epithelial ADAM17 has been shown to promote intestinal inflammation via the shedding of TNFa. Moreover, ADAM17 sheds JAM-A on epithelial cells, which is important for epithelial tight junction formation and thus for maintenance of the mucosal barrier ${ }^{17}$. Increased shedding of JAM-A driven by the iRhom2/ADAM17 axis may accelerate the inflammatory process. Of note, we observed a more pronounced effect of increased iRhom 2 expression on JAM-A shedding than TGFa shedding. This may be due to the different expression level and subcellular localization of the two substrates resulting in different susceptibility towards ADAM17 and different shedding kinetics. Although this may also indicate more potential long term relevance of JAM-A shedding it remains to be determined which shedding pathway is in fact contributing to disease development in vivo. Several more shedding events such as shedding of cadherins could also be implicated in this scenario. In fact, IFN $\gamma$ drives IBD pathogenesis through VE-cadherin-directed vascular barrier disruption ${ }^{66}$ and this could potentially be mediated by proteolytic shedding.

Because of its ambivalent role, general inhibition of ADAM17 does not appear to be reasonable in the treatment of chronic inflammatory diseases such as rheumatoid arthritis or IBD. As one potential strategy to limit the inflammatory activities more specifically and to spare physiological activities it has been suggested to develop iRhom2-specific inhibitors of ADAM17. These inhibitors would still allow iRhom1-dependent ADAM17 activity in non-inflamed tissue, for instance, growth factor driven regeneration. The findings of our present study suggest that induction of iRhom 2 in inflamed gut epithelial cells significantly contributes to the upregulated ADAM17 dependent mediator release. This upregulation pathway could be selectively prevented by iRhom 2 specific inhibitors allowing more selective targeting of inflammatory activities of ADAM17. 
A)

TGFa
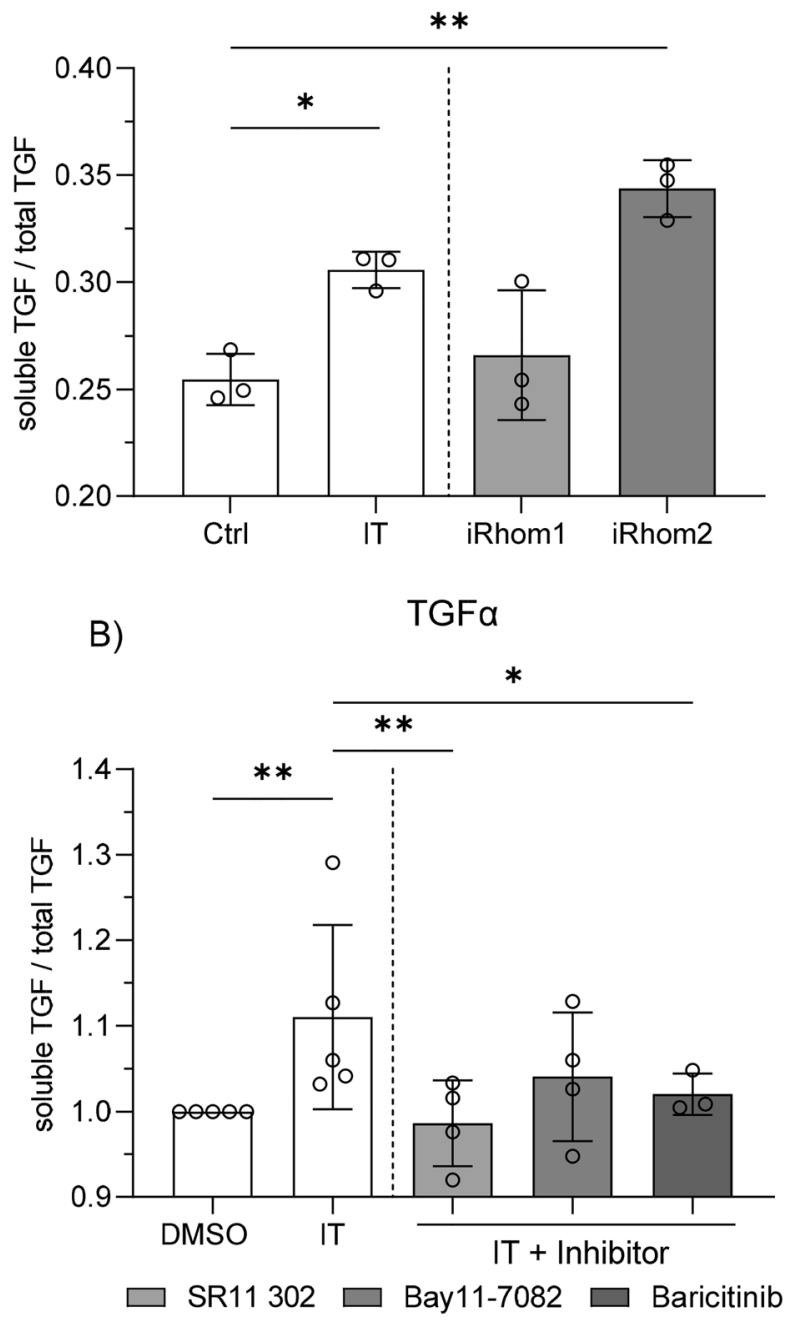

C)

Jam-A

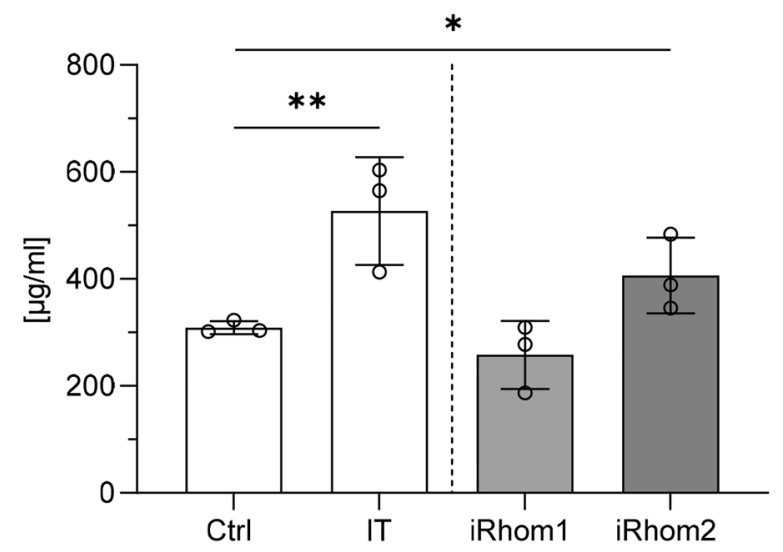


4Figure 6. Induction of ADAM17 mediated shedding by iRhom2 upregulation. (A): HT-29 cells transduced with lentivirus coding for iRhom 1 , iRhom 2 or control vector were transfected to express a TGF $\alpha$ alkaline phosphatase fusion protein. $24 \mathrm{~h}$ after transfection cells were either co-stimulated with IFN $\gamma$ and TNFa ((IT) each $10 \mathrm{ng} / \mathrm{ml}$ ) or left untreated. After another $24 \mathrm{~h}$ ADAM17 mediated shedding of the fusion protein was determined by a colorimetric assay for phosphatase activity. (B): Wildtype HT-29 cells were transfected to express a TGF $\alpha$ alkaline phosphatase fusion protein. $24 \mathrm{~h}$ after transfection cells were treated with the NF-kB inhibitor Bay11-7082 (3 $\mu \mathrm{M})$, the AP1 inhibitor SR11 $302(3 \mu \mathrm{M})$, the JAK inhibitor Baricitinib $(1 \mu \mathrm{M})$ or vehicle control (DMSO) for $1 \mathrm{~h}$ before starting the stimulation with IFN $\gamma$ and TNFa ((IT) each $10 \mathrm{ng} / \mathrm{ml}$ ). After 24 h, ADAM17 mediated shedding of TGFa-AP was determined by a colorimetric assay. (C): HT-29 cells transduced with lentivirus coding for iRhom1, iRhom 2 or control vector, either co-stimulated with IFN $\gamma$ and TNFa ((IT) each $10 \mathrm{ng} / \mathrm{ml}$ ) for $24 \mathrm{~h}$ or left untreated, were analyzed by ELISA for ADAM17 mediated release of cell expressed JAM-A.

\section{Data availability}

The original datasets generated or analyzed during the present study are available from the corresponding author on reasonable request.

Received: 4 August 2021; Accepted: 26 November 2021

Published online: 20 December 2021

\section{References}

1. Abdulkhaleq, L. A. et al. The crucial roles of inflammatory mediators in inflammation: A review. Vet World https://doi.org/10. 14202/vetworld.2018.627-635 (2018).

2. Ariel, A. \& Timor, O. Hanging in the balance: Endogenous anti-inflammatory mechanisms in tissue repair and fibrosis. J. Pathol. https://doi.org/10.1002/path.4108 (2013).

3. Eming, S. A., Krieg, T. \& Davidson, J. M. Inflammation in wound repair: Molecular and cellular mechanisms. J. Invest. Dermatol. https://doi.org/10.1038/sj.jid.5700701 (2007).

4. Barrientos, S., Stojadinovic, O., Golinko, M. S., Brem, H. \& Tomic-Canic, M. Growth factors and cytokines in wound healing. Wound Repair Regen. https://doi.org/10.1111/j.1524-475X.2008.00410.x (2008).

5. Peschon, J. J. et al. An essential role for ectodomain shedding in mammalian development. Science (80-). https://doi.org/10.1126/ science.282.5392.1281 (1998).

6. Schulz, B. et al. ADAMIO regulates endothelial permeability and T-cell transmigration by proteolysis of vascular endothelial cadherin. Circ Res. https://doi.org/10.1161/CIRCRESAHA.107.169805 (2008).

7. Hartmann, D. et al. The disintegrin/metalloprotease ADAM 10 is essential for Notch signalling but not for $\alpha$-secretase activity in fibroblasts. Hum. Mol Genet. https://doi.org/10.1093/hmg/11.21.2615 (2002).

8. Koenen, R. R. et al. Regulated release and functional modulation of junctional adhesion molecule A by disintegrin metalloproteinases. Blood https://doi.org/10.1182/blood-2008-04-152330 (2009).

9. Lee, D. C. et al. TACE/ADAM17 processing of EGFR ligands indicates a role as a physiological convertase. Ann. New York Acad. Sci. https://doi.org/10.1111/j.1749-6632.2003.tb03207.x (2003).

10. Pruessmeyer, J. et al. A Disintegrin and metalloproteinase 17 (ADAM17) mediates inflammation-induced shedding of syndecan-1 and -4 by lung epithelial cells. J Biol Chem. https://doi.org/10.1074/jbc.M109.059394 (2010).

11. Black, R. A. et al. A metalloproteinase disintegrin that releases tumour-necrosis factor- $\varnothing$ from cells. Nature https://doi.org/10. 1038/385729a0 (1997).

12. Moss, M. L. et al. Cloning of a disintegrin metalloproteinase that processes precursor tumour-necrosis factor- $\alpha$. Nature https:// doi.org/10.1038/385733a0 (1997).

13. Rosendahl, M. S. et al. Identification and characterization of a pro-tumor necrosis factor- $\alpha$ - processing enzyme from the ADAM family of zinc metalloproteases. J. Biol. Chem. https://doi.org/10.1074/jbc.272.39.24588 (1997).

14. Sahin, U. et al. Distinct roles for ADAM10 and ADAM17 in ectodomain shedding of six EGFR ligands. J. Cell Biol. https://doi.org/ $10.1083 /$ jcb.200307137 (2004).

15. Bamias, G., Corridoni, D., Pizarro, T. T. \& Cominelli, F. New insights into the dichotomous role of innate cytokines in gut homeostasis and inflammation. Cytokine https://doi.org/10.1016/j.cyto.2012.06.014 (2012).

16. Schultz, G., Clark, W. \& Rotatori, D. S. EGF and TGF- $\alpha$ in wound healing and repair. J. Cell Biochem. https://doi.org/10.1002/jcb. 240450407 (1991).

17. Laukoetter, M. G. et al. JAM-A regulates permeability and inflammation in the intestine in vivo. J. Cell Biol. https://doi.org/10. 1083/jcb1795oia14 (2007).

18. Chalaris, A. et al. Critical role of the disintegrin metalloprotease ADAM17 for intestinal inflammation and regeneration in mice. J. Cell Biol. https://doi.org/10.1083/jcb1901oia2 (2010).

19. Feng, Y. et al. Loss of ADAM17-mediated tumor necrosis factor alpha signaling in intestinal cells attenuates mucosal atrophy in a mouse model of parenteral nutrition. Mol. Cell Biol. https://doi.org/10.1128/mcb.00143-15 (2015).

20. Uhlen, M., Uhlén, M., Fagerberg, L., et al. Proteomics. Tissue-based map of the human proteome. Science (80- ). Published online 2015.

21. The Human Protein Atlas. Published online 2021. https://www.proteinatlas.org/ENSG00000151694-ADAM17

22. Yoda, M. et al. Systemic overexpression of TNFa-converting enzyme does not lead to enhanced shedding activity in vivo. PLoS ONE https://doi.org/10.1371/journal.pone.0054412 (2013).

23. Schlöndorff, J., Becherer, J. D. \& Blobel, C. P. Intracellular maturation and localization of the tumour necrosis factor a convertase (TACE). Biochem J. https://doi.org/10.1042/0264-6021:3470131 (2000).

24. Endres, K. et al. Tumor necrosis factor- $\alpha$ converting enzyme is processed by proprotein-convertases to its mature form which is degraded upon phorbol ester stimulation. Eur. J. Biochem. https://doi.org/10.1046/j.1432-1033.2003.03606.x (2003).

25. Adrain, C., Zettl, M., Christova, Y., Taylor, N. \& Freeman, M. Tumor necrosis factor signaling requires iRhom2 to promote trafficking and activation of TACE. Science https://doi.org/10.1126/science.1214400 (2012).

26. Christova, Y., Adrain, C., Bambrough, P., Ibrahim, A. \& Freeman, M. Mammalian iRhoms have distinct physiological functions including an essential role in TACE regulation. EMBO Rep. https://doi.org/10.1038/embor.2013.128 (2013).

27. Calligaris, M. et al. Strategies to target ADAM17 in disease: From its discovery to the iRhom revolution. Molecules https://doi.org/ 10.3390/molecules26040944 (2021).

28. Li, X. et al. iRhoms 1 and 2 are essential upstream regulators of ADAM17-dependent EGFR signaling. Proc Natl Acad Sci U S A. https://doi.org/10.1073/pnas.1505649112 (2015). 
29. Grieve, A. G. et al. Phosphorylation of iRhom 2 at the plasma membrane controls mammalian TACE-dependent inflammatory and growth factor signalling. Elife https://doi.org/10.7554/eLife.23968 (2017).

30. Cavadas, M. et al. Phosphorylation of iRhom 2 controls stimulated proteolytic shedding by the metalloprotease ADAM17/TACE. Cell Rep. https://doi.org/10.1016/j.celrep.2017.09.074 (2017).

31. Maretzky, T. et al. iRhom2 controls the substrate selectivity of stimulated ADAM17-dependent ectodomain shedding. Proc Natl Acad Sci U S A. https://doi.org/10.1073/pnas.1302553110 (2013).

32. Le Gall, S. M. et al. ADAM17 is regulated by a rapid and reversible mechanism that controls access to its catalytic site. J. Cell Sci. https://doi.org/10.1242/jcs.069997 (2010).

33. Grötzinger, J., Lorenzen, I. \& Düsterhöft, S. Molecular insights into the multilayered regulation of ADAM17: The role of the extracellular region. Biochim. Biophys. Acta Mol. Cell Res. https://doi.org/10.1016/j.bbamcr.2017.05.024 (2017).

34. Sommer, A. et al. Phosphatidylserine exposure is required for ADAM17 sheddase function. Nat. Commun. https://doi.org/10. 1038/ncomms11523 (2016).

35. Düsterhöft, S., Babendreyer, A., Giese, A. A., Flasshove, C. \& Ludwig, A. Status update on iRhom and ADAM17: It’s still complicated. Biochim Biophys. Acta Mol. Cell Res. https://doi.org/10.1016/j.bbamcr.2019.06.017 (2019).

36. Seifert, A. et al. The IRHOM2/ADAM17 axis attenuates bacterial uptake by phagocytes in a cell autonomous manner. Int. J. Mol. Sci. https://doi.org/10.3390/ijms21175978 (2020).

37. Chaohui, C. et al. iRhom2 promotes atherosclerosis through macrophage inflammation and induction of oxidative stress. Biochem Biophys. Res. Commun. https://doi.org/10.1016/j.bbrc.2018.07.133 (2018).

38. Lee, W. et al. IRhom1 regulates proteasome activity via PAC1/2 under ER stress. Sci. Rep. https://doi.org/10.1038/srep11559 (2015).

39. Zhou, Z. et al. Human rhomboid family-1 suppresses oxygen-independent degradation of hypoxia-inducible factor-1a in breast cancer. Cancer Res. https://doi.org/10.1158/0008-5472.CAN-13-1027 (2014).

40. Yan, Z. et al. Human rhomboid family-1 gene silencing causes apoptosis or autophagy to epithelial cancer cells and inhibits xenograft tumor growth. Mol. Cancer Ther. https://doi.org/10.1158/1535-7163.MCT-08-0104 (2008).

41. Gross, A. et al. Desmoglein 2, but not desmocollin 2, protects intestinal epithelia from injury. Mucosal. Immunol. https://doi.org/ 10.1038/s41385-018-0062-z (2018).

42. Hruz, T. et al. Genevestigator V3: a reference expression database for the meta-analysis of transcriptomes. Adv. Bioinform. https:// doi.org/10.1155/2008/420747 (2008).

43. Kent, W. J. et al. The human genome browser at UCSC. Genome Res. https://doi.org/10.1101/gr.229102 (2002).

44. Gearing, L. J. et al. CiIIder: A tool for predicting and analysing transcription factor binding sites. PLoS ONE https://doi.org/10. 1371/journal.pone.0215495 (2019).

45. Salmon, P. \& Trono, D. Production and Titration of Lentiviral Vectors. Curr. Protoc. Neurosci. https://doi.org/10.1002/0471142301. ns0421s37 (2006).

46. Babendreyer, A. et al. Differential induction of the ADAM17 regulators iRhom1 and 2 in endothelial cells. Front. Cardiovasc. Med. https://doi.org/10.3389/fcrm.2020.610344 (2020).

47. Inoue, A. et al. TGFa shedding assay: An accurate and versatile method for detecting GPCR activation. Nat. Methods. https://doi. org/10.1038/nmeth.2172 (2012).

48. Obermeier, F. et al. Interferon-gamma (IFN- $\gamma$ )- and tumour necrosis factor (TNF)-induced nitric oxide as toxic effector molecule in chronic dextran sulphate sodium (DSS)-induced colitis in mice. Clin. Exp. Immunol. https://doi.org/10.1046/j.1365-2249.1999. 00878.x (1999).

49. Mcllwain, D. R. et al. iRhom2 regulation of TACE controls TNF-mediated protection against Listeria and responses to LPS. Science https://doi.org/10.1126/science.1214448 (2012).

50. Siggs, O. M. et al. iRhom2 is required for the secretion of mouse TNFa. Blood https://doi.org/10.1182/blood-2012-03-417949 (2012).

51. Issuree, P. D. A. et al. iRHOM2 is a critical pathogenic mediator of inflammatory arthritis. J. Clin. Invest. https://doi.org/10.1172/ JCI66168 (2013).

52. Liu, T., Zhang, L., Joo, D. \& Sun, S. C. NF-kB signaling in inflammation. Signal Transduct. Target Ther. https://doi.org/10.1038/ sigtrans.2017.23 (2017).

53. Lin, Y., Jamison, S. \& Lin, W. Interferon- $\gamma$ activates nuclear factor- $\kappa$ B in oligodendrocytes through a process mediated by the unfolded protein response. PLoS ONE https://doi.org/10.1371/journal.pone.0036408 (2012).

54. Thapa, R. J. et al. NF-B protects cells from gamma interferon-induced RIP1-dependent necroptosis. Mol Cell Biol. https://doi.org/ 10.1128/mcb.05445-11 (2011).

55. Shimoda, M. et al. Epithelial cell-derived a disintegrin and metalloproteinase-17 confers resistance to colonic inflammation through EGFR activation. EBioMedicine https://doi.org/10.1016/j.ebiom.2016.02.007 (2016).

56. Dulloo, I., Muliyil, S. \& Freeman, M. The molecular, cellular and pathophysiological roles of irhom pseudoproteases. Open Biol. https://doi.org/10.1098/rsob.190003 (2019).

57. Hu, S. et al. TNF- $\alpha$ and IFN- $\gamma$ synergistically inhibit the repairing ability of mesenchymal stem cells on mice colitis and colon cancer. Am. J. Transl. Res. 11, 6207 (2019).

58. O'Connell, D. et al. IFN- $\gamma$-induced JAK/STAT, but not NF-kb, signaling pathway is insensitive to glucocorticoid in airway epithelial cells. Am. J. Physiol. Lung Cell Mol. Physiol. https://doi.org/10.1152/ajplung.00099.2015 (2015).

59. Gough, D. J., Levy, D. E., Johnstone, R. W. \& Clarke, C. J. IFN $\gamma$ signaling-Does it mean JAK-STAT?. Cytokine Growth Factor Rev. https://doi.org/10.1016/j.cytogfr.2008.08.004 (2008).

60. Lowenthal, J. W., Ballard, D. W., Bohnlein, E. \& Greene, W. C. Tumor necrosis factor a induces proteins that bind specifically to кB-like enhancer elements and regulate interleukin 2 receptor $\alpha$-chain gene expression in primary human T lymphocytes. Proc. Natl. Acad. Sci. USA. https://doi.org/10.1073/pnas.86.7.2331 (1989).

61. Osborn, L., Kunkel, S. \& Nabel, G. J. Tumor necrosis factor $\alpha$ and interleukin 1 stimulate the human immunodeficiency virus enhancer by activation of the nuclear factor кB. Proc. Natl. Acad. Sci. USA. https://doi.org/10.1073/pnas.86.7.2336 (1989).

62. Matsumiya, T. et al. Characterization of synergistic induction of CX3CL1/Fractalkine by TNF- $\alpha$ and IFN- $\gamma$ in vascular endothelial cells: an essential role for TNF- $\alpha$ in post-transcriptional regulation of CX3CL1. J. Immunol. https://doi.org/10.4049/jimmunol. 0903212 (2010).

63. Geesala, R. et al. Loss of RHBDF2 results in an early-onset spontaneous murine colitis. J. Leukoc Biol. 105(4), 767-781. https:// doi.org/10.1002/JLB.4A0718-283RR (2019).

64. Luo, W. W. et al. iRhom 2 is essential for innate immunity to RNA virus by antagonizing ER- and mitochondria-associated degradation of VISA. PLoS Pathog. https://doi.org/10.1371/journal.ppat.1006693 (2017).

65. Koff, J. L., Shao, M. X. G., Kim, S., Ueki, I. F. \& Nadel, J. A. Pseudomonas lipopolysaccharide accelerates wound repair via activation of a novel epithelial cell signaling cascade. J. Immunol. https://doi.org/10.4049/jimmunol.177.12.8693 (2006).

66. Langer, V. et al. IFN- $\gamma$ drives inflammatory bowel disease pathogenesis through VE-cadherin-directed vascular barrier disruption. J. Clin. Invest. https://doi.org/10.1172/JCI124884 (2019).

\section{Acknowledgements}

We thank T. Woopen for expert technical assistance. 


\section{Author contributions}

A.A.G., A.B., S.D. and A.L. designed the study; A.A.G. and P.K. performed experiments; A.G. and P.S. provided tissues from murine models, A.B. and S.D. analyzed data; A.A.G. and A.L. and wrote the manuscript; all authors reviewed the manuscript.

\section{Funding}

Open Access funding enabled and organized by Projekt DEAL. This work was supported in part by the German Research Foundation DFG Grant Lu869/8-1 to AL; ERS Start-up (StUpPD_299-18) and START Grant (\#691903-06/19) to SD.

\section{Competing interests}

The authors declare no competing interests.

\section{Additional information}

Supplementary Information The online version contains supplementary material available at https://doi.org/ 10.1038/s41598-021-03522-2.

Correspondence and requests for materials should be addressed to A.L.

Reprints and permissions information is available at www.nature.com/reprints.

Publisher's note Springer Nature remains neutral with regard to jurisdictional claims in published maps and institutional affiliations.

(c) (i) Open Access This article is licensed under a Creative Commons Attribution 4.0 International License, which permits use, sharing, adaptation, distribution and reproduction in any medium or format, as long as you give appropriate credit to the original author(s) and the source, provide a link to the Creative Commons licence, and indicate if changes were made. The images or other third party material in this article are included in the article's Creative Commons licence, unless indicated otherwise in a credit line to the material. If material is not included in the article's Creative Commons licence and your intended use is not permitted by statutory regulation or exceeds the permitted use, you will need to obtain permission directly from the copyright holder. To view a copy of this licence, visit http://creativecommons.org/licenses/by/4.0/.

(C) The Author(s) 2021 